\title{
Lateral Thyrotomy Approach in the Era of Transoral Laser Surgeries
}

\author{
BV Manjula ${ }^{1}$, R Kamala $^{2}$, R Vaishnavi $^{3}$, Brinda A Poojari ${ }^{4}$
}

\begin{abstract}
Background: Laryngeal lipomas are rare benign lesions and are usually asymptomatic till they grow in size to become obstructive. Excision of a laryngeal submucosal lesion presents with the dilemma of an external or an endoscopic approach. The transoral endoscopic approach is preferred for smaller lesions and external approach for large tumors. This report introduces the external lateral thyrotomy approach for excision of a paraglottic space lipoma.

Case description: A 33-year-old female patient, teacher by profession, presented with complaints of voice change and choking sensation for 3 months. Flexible laryngoscopy revealed a smooth submucosal swelling of the right false cord and aryepiglottic (AE) fold with fullness in the right pyriform fossa and bilateral mobile vocal cords. Contrast-enhanced computed tomography (CECT) scan of neck was suggestive of a right paraglottic $5 \times 3 \mathrm{~cm}$ dumbbell-shaped lipoma that was excised by the lateral thyrotomy approach.

Conclusion: The lateral thyrotomy approach provided adequate exposure of the tumor and excellent visibility of the entire paraglottic space under direct vision. A complete excision could be performed without any breach of the laryngeal mucosa or tracheostomy and with preservation of voice and laryngeal framework.

Clinical significance: An individualized approach is needed for rare tumors and sometimes a simple procedure may outweigh the benefits of an advanced technique.

Keywords: Benign laryngeal tumor, Hoarseness, Laryngeal framework, Laryngeal lipoma, Laryngeal neoplasm, Lateral thyrotomy, Lipoma. International Journal of Phonosurgery \& Laryngology (2020): 10.5005/jp-journals-10023-1187
\end{abstract}

\section{INTRODUCTION}

Laryngeal lipomas are rare benign lesions comprising $0.6 \%$ of all benign laryngeal tumors and $1 \%$ of all benign lipomas. ${ }^{1}$ They originate from parts of larynx that normally contain fat and can be classified into extrinsic and intrinsic tumors. Tumors located in the posterior aspect of the larynx, pyriform sinus, and lingual surface of the epiglottis are extrinsic lipomas and those located in the false vocal cord, laryngeal surface of epiglottis, aryepiglottic (AE) folds, paraglottic space, and subglottic larynx are intrinsic lipomas. ${ }^{2}$ Clinically, lipoma presents as painless, smooth, well-defined, or pedunculated submucosal mass usually involving one side of the larynx, obliterating the AE fold, bulging over the lumen of the larynx, and obscuring the true vocal cord. ${ }^{3}$ They can be asymptomatic or have nonspecific symptoms like paroxysmal coughing, choking, hoarseness of voice, and dysphagia. In later stages, however, the patient may present with dyspnea and stridor, because of the bulky tumor mass causing airway obstruction. ${ }^{4}$

Excision of a paraglottic space submucosal lesion presents with the dilemma of an external or an endoscopic approach. The transoral endoscopic approach is preferred for smaller lesions and the external approach for large tumors. Lateral thyrotomy is an age-old approach that was first proposed by Lewis in 1914, for congenital cyst dissection under direct vision, where a vertical split was made through the thyroid lamina just anterior to the superior cornu. This approach was later modified by many but has been widely used for laryngocele excision and occasionally for other benign tumors of paraglottic space. ${ }^{5}$ This current case report proposes the use of the lateral thyrotomy approach for complete excision of paraglottic space submucosal lipoma with preservation of voice and laryngeal framework without a tracheostomy.

\begin{abstract}
${ }^{1-4}$ Department of ENT and Head and Neck Surgery, Bengaluru Baptist Hospital, Bengaluru, Karnataka, India

Corresponding Author: BV Manjula, Department of ENT and Head and Neck Surgery, Bengaluru Baptist Hospital, Bengaluru, Karnataka, India, Phone: +91 9980166444, e-mail: drmanjubv@gmail.com

How to cite this article: Manjula BV, Kamala R, Vaishnavi R, et al. Lateral Thyrotomy Approach in the Era of Transoral Laser Surgeries. Int J Phonosurg Laryngol 2020;10(2):58-61.
\end{abstract}

Source of support: Nil

Conflict of interest: None

\section{Case Description}

A 33-year-old lady, teacher by profession, presented to us with complaints of voice change and choking sensation for past 3 months. She had no other comorbidities or habits. Flexible laryngoscopy revealed a smooth submucosal swelling of the right false cord and aryepiglottic fold with fullness in right pyriform fossa and bilateral mobile vocal cords (Fig. 1). She underwent contrast-enhanced computed tomography (CECT) scan neck, which was suggestive of a well-circumscribed $5 \times 3 \mathrm{~cm}$ lesion in right paraglottic space with extralaryngeal extension and with a very low radiodensity highly suggestive of lipoma (Fig. 2). As she is a professional voice user, an external approach was preferred to avoid trauma and scarring of endolarynx.

\section{Surgical Procedure}

A schematic representation of the surgical procedure is shown in Figure 3. Following a routine horizontal neck crease incision, subplatysmal flaps elevated, strap muscles were cut superiorly 

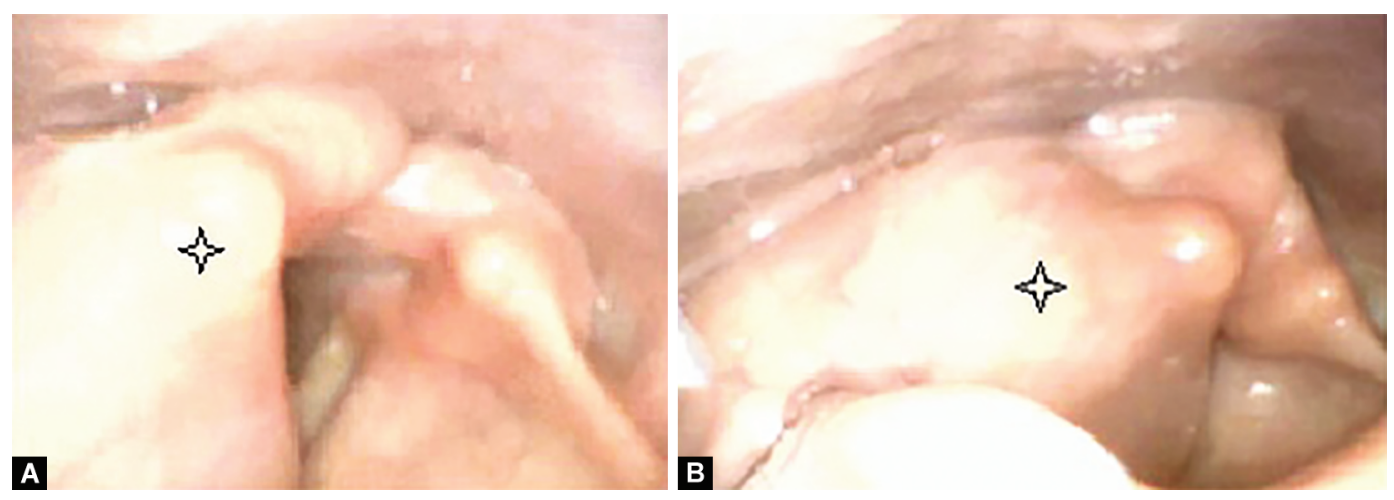

Figs $1 \mathrm{~A}$ and B: Preoperative videolaryngoscopic image of right laryngeal submucosal bulge involving right aryepiglottic fold and false cord
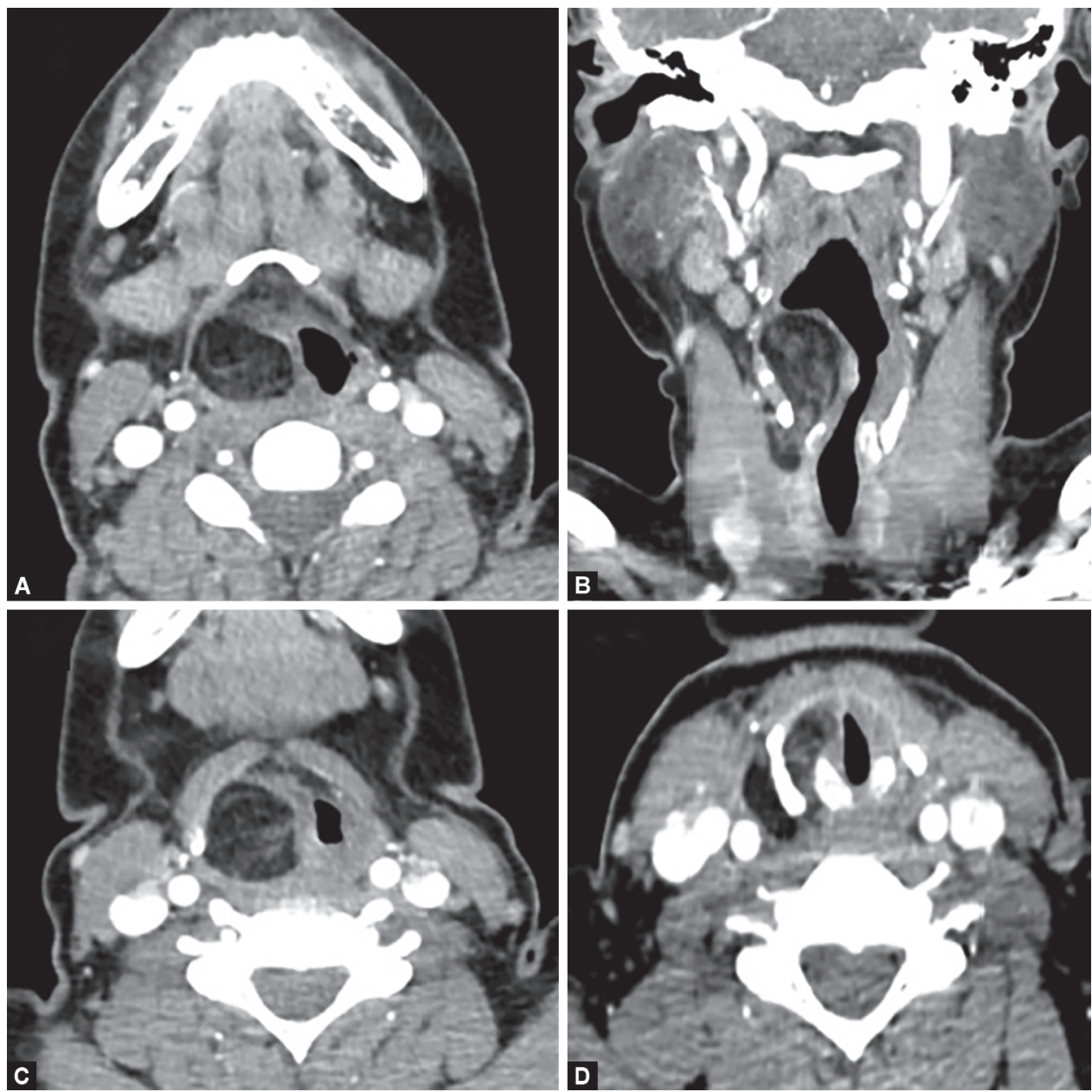

Figs $2 \mathrm{~A}$ to D: Preoperative contrast-enhanced computed tomography images showing a $5 \times 3 \mathrm{~cm}$ right paraglottic lesion with extralaryngeal extension

close to their insertion and thyroid cartilage was exposed. Thyroid notch, anterior and inferior borders were identified. Perichondrium was elevated and cartilage was spilt vertically in the junction of anterior one-third and posterior two-third and retracted (Figs 4A to C). Retraction of thyroid lamina provided adequate exposure of the tumor and excellent visibility of entire paraglottic space under direct vision for complete excision. The tumor was separated using blunt dissection all around and both intra- and extralaryngeal parts were removed in toto. There was no breach in the laryngeal mucosa. Histopathology confirmed the lesion to be lipoma.

The hoarseness in her voice improved immediately during the postoperative period. Flexible laryngoscopy performed on day 1 and day 20 postoperatively showed no edema of the laryngeal inlet. Bilateral vocal cords were normal and mobile and glottic chink was normal (Fig. 4D). Since lipomas are known to recur, 


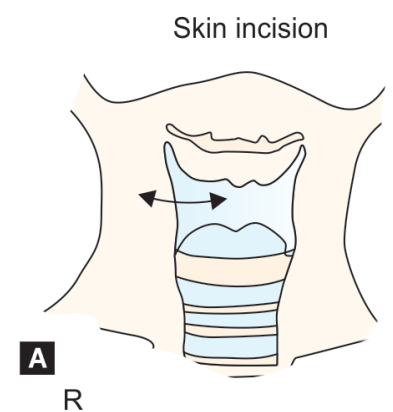

$R$

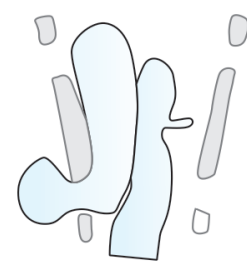

$\mathrm{R}$

C

Extralaryngeal extension
$\mathrm{L}$

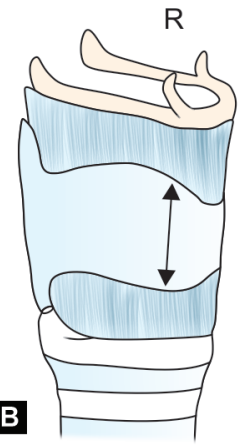

D

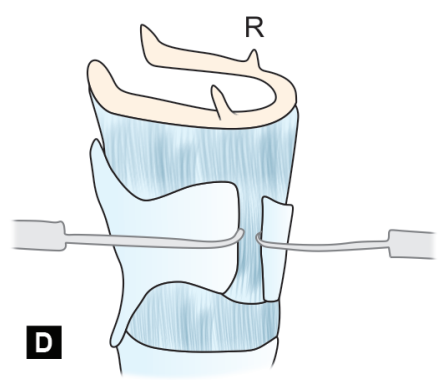

Figs $3 A$ to $D$ : Schema of right lateral thyrotomy (R, right; $L$, left) she was followed up for a period of 1 year and found to have no recurrence.

\section{Discussion}

Paraglottic space in the larynx lies on either side of glottis, bounded medially by the quadrangular membrane, ventricle, and conus elasticus; laterally by the thyroid cartilage and cricothyroid membrane; and posteriorly by the mucosa of the piriform sinus. This space encompasses the laryngeal ventricle and saccule, and it communicates anterosuperiorly with the pre-epiglottic space. At the level of supraglottic larynx, the paraglottic space is filled mostly with fat and below the ventricle; the thyroarytenoid muscle fills this space. This space is continuous anterolaterally with the extralaryngeal soft tissue between the thyroid and cricoid cartilages - an important pathway for extralaryngeal tumor spread. Paraglottic space is a common site for various laryngeal submucosal lesions like schwannoma, laryngocele, lipoma, liposarcoma, oncocytoma, granular cell tumor, hemangioma, and adenoma. ${ }^{6}$

Due to its rarity, opinion is divided and laryngeal lipomas have no particular management protocol. A customized management plan is required for each patient based on the symptoms and extent of the tumor. The two approaches available are external and internal. The internal approach involves the use of carbon dioxide laser $\left(\mathrm{CO}_{2}\right)$ for excision under microscopic or direct video
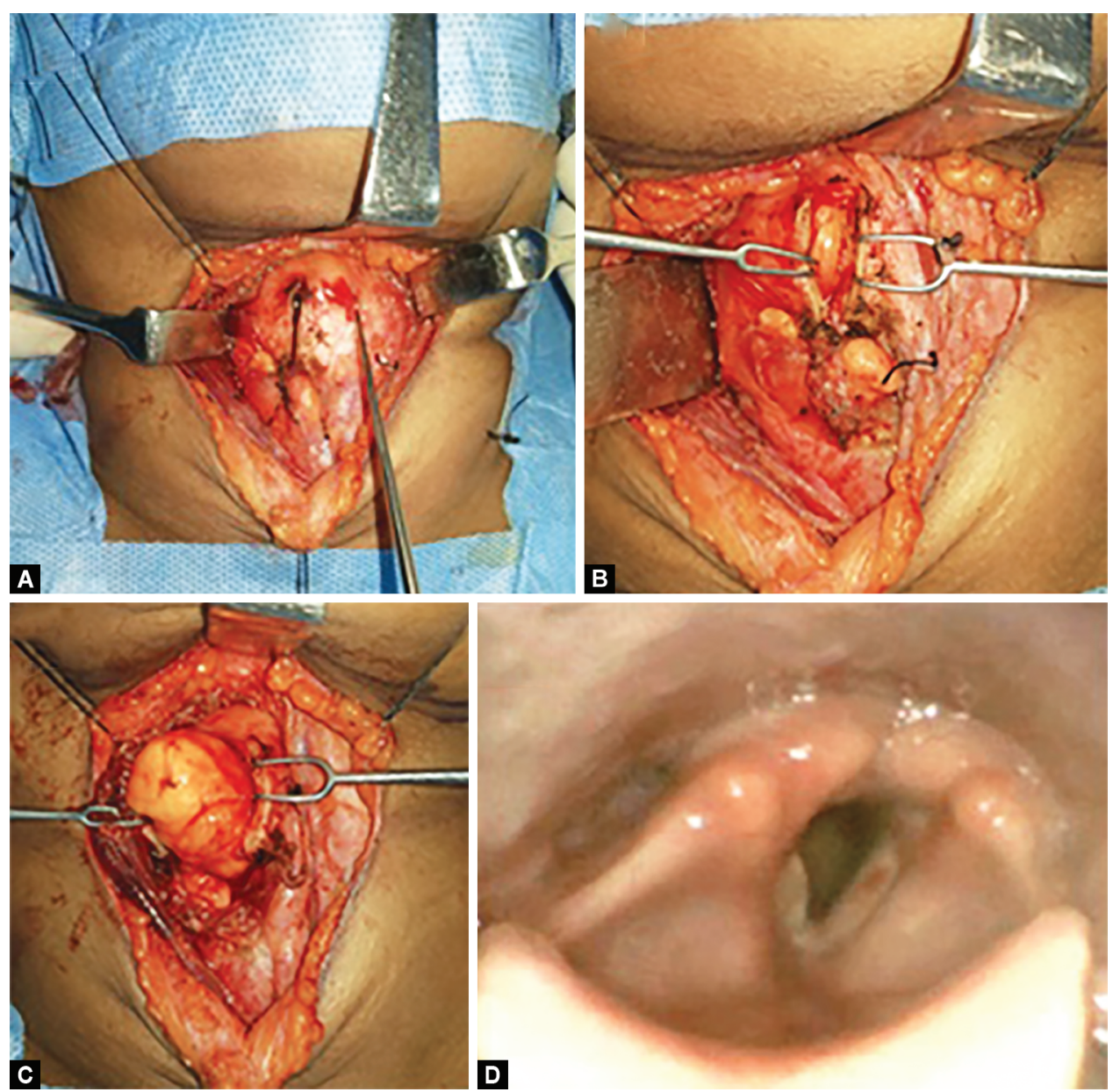

Figs 4A to D: (A) Vertical thyrotomy; (B) Split thyroid lamina retracted; (C) Lipoma dissected free from paraglottic space; (D) Postoperative laryngoscopy showing normal endolarynx 
laryngoscopic vision. Microlaryngeal surgery (MLS) along with $\mathrm{CO}_{2}$ laser is now the procedure of choice for benign, premalignant, or malignant lesions of the larynx in early stages. It is quicker, more precise, and safer alternative with fewer complications than external approaches. ${ }^{7}$ There are limitations for MLS in case of larger lesions, as it provides a limited surgical exposure, which may lead to incomplete excision of the lesion and hence increase the probability of recurrence. It also causes mucosal trauma and endolaryngeal scarring. Also a microflap needs to be created, before resection of submucosal lesion in order to protect the vocal ligament, due to the high content of fibroblast in the lamina propria, which predisposes it to major scarring. ${ }^{8}$ Other drawbacks include trauma to neighboring laryngeal structures with intraoperative complication rate of $14.8 \%$ like severe bradycardia, oral cavity/pharyngeal wall bleeding, severe dyspnea postextubation, cervical emphysema, tooth damage, cuff damage during $\mathrm{CO}_{2}$ laser, and fire accidents. In case of larger lesions where access or manipulation maybe difficult, an external approach can provide wider field. ${ }^{7}$

In 1914, Lewis ${ }^{5}$ first introduced the lateral thyrotomy approach on the paraglottic space for congenital laryngeal cyst dissection under direct vision through a vertical split of the thyroid lamina just anterior to the superior cornu. It has been modified over the years by many surgeons for excision of cysts and other benign laryngeal tumors. Some have recommended removal of a small portion of the thyroid cartilage also to provide sufficient exposure whereas others have adopted a more conservative approach. ${ }^{9}$

Advantages of this external approach include no breach of the laryngeal mucosa when meticulous dissection is done, avoidance of tracheostomy and scarring due to laryngeal mucosal trauma. The major portion of paraglottic space contains only fat, which allows tumors to grow considerably without causing symptoms and there are no important neural or vascular structures in the paraglottic space. Most of these tumors remain lateral to the quadrangular membrane, which limit their extension into the supraglottic mucosa. Many authors have even proposed that external thyrotomy is possible under local anesthesia to excise these lesions. ${ }^{8,9} \mathrm{~A}$ suprathyroid alar cartilage approach has also been described for such submucosal lesions where access can be gained by retraction of thyroid lamina anteroinferiorly without any distortion of the laryngeal framework. ${ }^{10}$

The lateral thyrotomy approach has proven to provide good exposure of paraglottic space for safe dissection of lesions like laryngoceles. It has the advantage of direct access to paraglottic space ensuring precise dissection under direct vision for submucosal resection. It also minimizes trauma to neighboring laryngeal structures and allows the surgeon to ascertain complete removal, thereby preventing recurrence. The untouched endolarynx results in the preservation of the mucosal anatomy. The laryngeal framework is not deformed; only a split in thyroid lamina is made to gain a wide operative field that is used for a more precise surgical technique..$^{6,8,9}$
Very little has been reported on an external approach via lateral thyrotomy for other benign laryngeal lesions and the potential of this approach has not been appreciated.

\section{Conclusion}

Treatment of benign laryngeal lesions should employ a conservative surgical approach. Lateral thyrotomy approach was found to be best suited in this patient as it was a large paraglottic lipoma with an extralaryngeal component. Profession of the patient and the submucosal nature of the lesion required no breach of the laryngeal mucosa and this was achieved in the external approach. Also it avoided the risk of airway edema and need for tracheostomy. The lateral thyrotomy approach thus made it possible for complete tumor removal with preservation of voice and the laryngeal framework. It can be considered a safe and useful surgical option for benign paraglottic submucosal lesions even in this era of minimally invasive transoral laser surgeries.

\section{Declaration of Patient Consent}

The authors certify that they have obtained an appropriate patient consent form.

\section{References}

1. El-Monem MHA, Gaafar AH, Magdy EA. Lipomas of the head and neck: presentation variability and diagnostic work-up. J Laryngol Otol 2006;120(1):47-55. DOI: 10.1017/S0022215105004597.

2. Zakrzewski A. Subglottic lipoma of the larynx. (case report and literature review). J Laryngol Otol 1965;79(12):1039-1048. DOI: $10.1017 /$ S0022215100064781.

3. Wenig BM. Lipomas of the larynx and hypopharynx: a review of the literature with the addition of three new cases. J Laryngol Otol 1995;109(4):353-357. DOI: 10.1017/S0022215100130130.

4. Kodiyan J, Rudman JR, Rosow DE, et al. Lipoma and liposarcoma of the larynx: case reports and literature review. Am J Otolaryngol 2015;36(4):611-615. DOI: 10.1016/j.amjoto.2014.07.003.

5. Lewis DD. Discussion on ventricle of larynx. Ann Otol Rhinol Laryngol 1914;24:129-138.

6. Benninger MS, Sataloff RT. Benign tumors of the larynx. Sataloff's comprehensive textbook of otolaryngology head and neck surgery. Laryngology 2016;4(36-37):757-768.

7. Chiesa-Estomba CM, González-García JA, Larruscain E, et al. CO2 transoral laser microsurgery in benign, premalignant and malignant ( $\mathrm{Tis}, \mathrm{T} 1, \mathrm{~T} 2)$ lesion of the glottis. A literature review. Medicines (Basel) 2019;6(3):77. DOI: 10.3390/medicines6030077.

8. Thomé R, Thomé DC, De La Cortina RA. Lateral thyrotomy approach on the paraglottic space for laryngocele resection. Laryngoscope 2000;110(3 Pt 1):447-450. DOI: 10.1097/00005537-200003000-00023.

9. Myssiorek D, Madnani D, Delacure MD. The external approach for submucosal lesions of the larynx. Otolaryngol Neck Surg 2001;125(4):370-373. DOI: 10.1067/mhn.2001.118690.

10. Ueha R, Nito T, Sakamoto $T$, et al. Supra-thyroid alar cartilage approach for the complete resection of laryngeal submucosal tumors and postoperative voice quality. Eur Arch Otorhinolaryngol 2015;272(10):2907-2913. 